\title{
Evaluation of Labor Burden Reduction Achieved through Wearing an Agricultural Power Assist Suit
}

\author{
Toshitake Araie, Uichi Nishizawa, Tomozumi Ikeda, Akira Kakimoto, and Shigeki Toyama
}

\begin{abstract}
This study develops a power assist suit (PAS) to assist laborers in agricultural work. This device is expected to reduce the strain produced by heavy agricultural work. During grape cultivation, pruning fruits in a posture, where workers have to keep their arms raised continuously, imposes a heavy burden on the workers including elderly. The objective of the PAS is to reduce the fatigue involved in the fruit pruning task by mounting a motor and spring-assisted mechanism on the suit. Quantifying the degree of fatigue reduction during agricultural work was necessary while developing the PAS. In this study, the surface electromyography (EMG), amount of energy expended, and exercise intensity were measured for motions similar to those involved in the shelf work in grape cultivation to verify the effects of reducing labor while wearing a PAS for agricultural work. The results demonstrated that the PAS developed for agricultural work could effectively reduce labor during static motions, such as maintaining postures.
\end{abstract}

Index Terms-Power assist suit, agricultural work, surface EMG, energy expenditure, heart rate reserve.

\section{INTRODUCTION}

In recent years, efforts have been made to mechanize agricultural work to lighten the burden on agricultural workers. The benefits of agricultural mechanization appear most quickly in rice cultivation and animal husbandry, among others; therefore, mechanization was introduced for practical applications in these cases. However, the machinery for these applications is mainly large. Hence, its use is limited to broad, flat land. Mechanization has not yet progressed as far for these applications because horticulture and other branches of agriculture require delicate manual work in farm fields that are small and often sloped.

In view of this background, many institutions are researching assist suits that agricultural workers can wear to reduce their workload. Examples of these include the Raku Vest ARM-1 developed by Kubota Corporation and the WAS-LiBERo [1], [2] developed at Wakayama University. The former is for light work, such as shelf crop harvesting, while the latter includes two types: one for light operations, and another for heavy operations, such as lifting heavy loads. However, a single assist suit cannot be used to perform both light and heavy work.

Accordingly, interview surveys were conducted at the grape farms in Yamanashi Prefecture to research on the application of a robot suit developed for medical

Manuscript received January 23, 2017; revised April 15, 2017.

T. Araie, T. Ikeda, and A. Kakimoto are with the Polytechnic University of Japan, 2-32-1, Ogawa-nishimachi, Kodaira-shi, Tokyo, Japan (e-mail: araie@uitec.ac.jp,ikeda@uitec.ac.jp,kakimoto@uitec.ac.jp).

U. Nishizawa and S. Toyama are with the Tokyo University of Agriculture and Technology, 2-24-16, Nakacho, Koganei, Tokyo, Japan (e-mail: n-uichi@cc.tuat.ac.jp, toyama@cc.tuat.ac.jp). rehabilitation to agricultural use. The results showed that a great load is placed on the arms and hips during gathering or pruning of agricultural products, and assistance for the worker's posture is necessary [3]-[5].

In view of the above, a power assist suit (i.e., Agricultural PAS) that can support the same stance for long periods of time and includes a power assist unit that aids in the transport of harvested items and other heavy work has been developed to reduce the work load on agricultural workers. The quantification and evaluation of the wearer's fatigue level are important for the development of the Agricultural PAS. Therefore, the measurements of the surface muscle potential, amount of energy consumption, and motion strength during actions that simulated shelf work in this study were made both when the Agricultural PAS was and was not worn. Similar measurements were also performed for actions simulating motion in farm fields. These fatigue evaluations were used to verify the labor reducing effects of the Agricultural PAS.

\section{AgRICULTURAL PAS}

\section{A. Summary of the Agricultural PAS}

The Agricultural PAS used in this study was developed to reduce the workload for agricultural workers cultivating grapes, Asian pears, and other shelf crops. The suit maintains the agricultural worker's stance by spring or ratchet mechanisms without the use of motors or batteries.

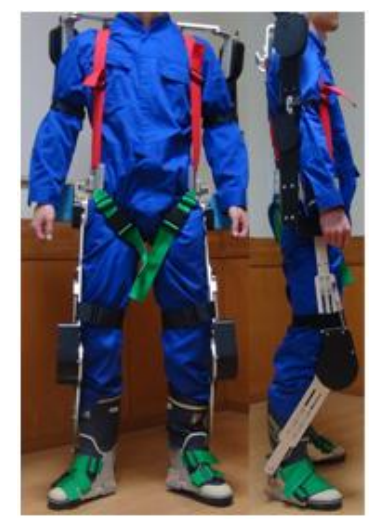

Previous Suit

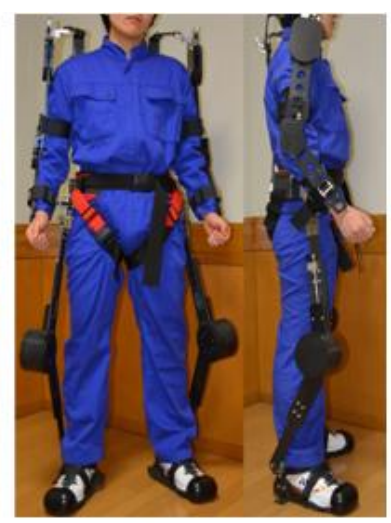

Current Suit
Fig. 1. Agricultural power assist suit.

Fig. 1 shows an outer view of the Agricultural PAS used in the tests. A previous suit is shown on the left, whereas the current suit is shown on the right. Both units assist in light work by maintaining the arms that handle the load in shelf crop harvesting in a raised stance and the hips in a half-sitting posture. The suit is structured such that the wearer does not feel the weight of the unit while in a standing or half-sitting 
posture. The design standard was set close to the average build of a male Japanese person with a height of $170 \mathrm{~cm}$ and a weight of $65 \mathrm{~kg}$. The frame position of each part can be adjusted to change the size. The time required for putting it on is approximately $1 \mathrm{~min}$.

\section{B. Previous Agricultural PAS}

The frame with an exoskeletal structure was made of A2017 aluminum having a total weight of approximately 16 $\mathrm{kg}$. The fixed positions with the body were the hips, arms, thighs, and feet, which a single operator can wear. A ratchet mechanism was incorporated into the shoulder joint area to hold the wearer's arms. The ratchet mechanism's assist force for each arm with an allowable ratchet torque of $29.4 \mathrm{Nm}$ and an arm length of $0.24 \mathrm{~m}$ from the ratchet center was $122.5 \mathrm{~N}$, with the total for both arms being $245 \mathrm{~N}$. In addition, a spiral spring was attached to the knee joint. The reaction force of this spiral spring assisted the wearer in a half-sitting posture. The spring mechanism assist force that results from the spring constant of $0.82 \mathrm{Nm} / \mathrm{deg}$ and the maximum usage angle of $120^{\circ}$ has a maximum torque of $98.64 \mathrm{Nm}$. With a frame length of $0.41 \mathrm{~m}$ from the knee center to the knee joint, it becomes $240.59 \mathrm{~N}$, and the total for both feet is $481.18 \mathrm{~N}$. The spring mechanism was activated using a switch installed in the thigh frame, enabling the wearer to activate the assist from any position.

\section{Current Agricultural PAS}

Fig. 2 shows the current suit assist mechanism. The frame material in the current suit was changed to carbon fiber-reinforced plastic to lighten the weight, reaching a total mass of approximately $8 \mathrm{~kg}$. The knee was not fixed, but set in a free state, to increase the body's freedom of movement. With this mechanism, the limitations on the knee joint flexion and on the lower limb inner and outer rotations were reduced, enabling smooth walking, change of direction, and lower body twisting movement. A plunger was used in the ratchet mechanism switch, and the shoulder abduction action enabled operation of this plunger. Hence, the assist can be switched on and off even if both hands are occupied. In addition to the assist mechanism for light work, a power assist unit for aid in lifting was included to further enable assistance for heavy work.

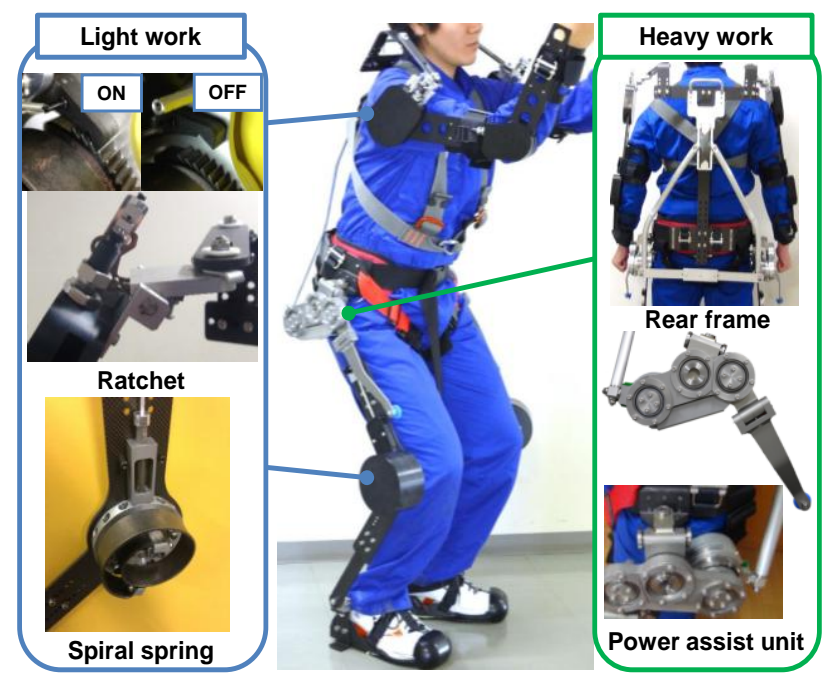

Fig. 2. Assist mechanism of corrent suit.
The power assist unit aids in heavy work, such as transporting agricultural crops. This unit can be mounted lateral to the current suit hip frame.

\section{EVALUATION INDICES}

\section{A. Surface Electromyography (EMG)}

The electromyogram frequency band falls to a lower frequency and the drop in the mean power frequency (MPF) $[\mathrm{Hz}]$ becomes a simple index for muscle fatigue when the muscle is fatigued [6]. The MPF was provided as follows, where $f$ is the frequency; $P(f)$ is the power spectrum of the muscle potential signal; and $f l$ to $f h$ is the frequency analysis section for the myoelectric signal:

$$
M P F=\frac{\int_{f l}^{f h} f \cdot P(f) d f}{\int_{f l}^{f h} P(f) d f}
$$

In addition, the muscle potential and the muscle force in the isometric movement that is not accompanied by articular movement show a generally linear relationship. In this study, the muscle force during maximum voluntary contraction (hereafter, MVC) is expressed as a ratio (\%MVC [\%]) to the amplitude of the muscle potential to be used as an index for the degree of the muscle load. The following formula was used to calculate \%MVC with the mean value of the amplitude of the muscle potential during operations given by AMP [V] and the mean value of the amplitude of the muscle potential during $\mathrm{MVC}$ given by $\mathrm{AMP}_{\mathrm{MVC}}[\mathrm{V}]$ :

$$
\% M V C=\frac{A M P}{A M P_{M V C}} \times 100
$$

\section{B. Amount of Energy Consumption and Motion Strength}

Humans use red blood cells in the bloodstream to send oxygen to the mitochondria within the body cells and use this oxygen to burn glucose and provide the energy required for life activities [7]. More energy is produced when a person is fatigued. Hence, more oxygen is needed, and the volume of the carbon dioxide discharged increases [8]. The energy expenditure (EE) [kcal/day] was obtained as follows from the oxygen intake volume $\left(\mathrm{VO}_{2}[\mathrm{ml} / \mathrm{min}]\right)$ and carbon dioxide discharge volume $\left(\mathrm{VCO}_{2}[\mathrm{ml} / \mathrm{min}]\right)$ :

$$
E E=\left(3.9 \times V O_{2}-1.1 \times V C O_{2}\right) \times 1.44
$$

The following formula can be used to determine the heart rate reserve (HRR) [\%] from the heart rate (HR), heart rate at rest $\mathrm{HR}_{\text {rest }}$, and maximum heart rate $\mathrm{HR}_{\text {max }}$ :

$$
H R R=\frac{H R-H R_{\text {rest }}}{H R_{\max }-H R_{\text {rest }}}
$$

\section{VERIFICATION OF REDUCING LABOR EFFECTS}

The surface muscle potential, amount of energy consumption, and motion strength evaluation indices were 
used to verify the labor-reducing effects of using the Agricultural PAS when performing light work.

TABLE I: PHYSICAL CHARACTERISTICS OF SUBJECTS

\begin{tabular}{ccccccccc}
\hline \hline & A & B & C & D & E & F & G & H \\
\hline Sex & male & male & male & male & female & male & female & male \\
Age & 22 & 22 & 22 & 22 & 49 & 69 & 48 & 72 \\
Height $(\mathrm{cm})$ & 169 & 172 & 175 & 168 & 167 & 165 & 156 & 153 \\
Weight $(\mathrm{kg})$ & 55 & 58 & 65 & 64 & 55 & 50 & 53 & 54 \\
Resting heart rate $(\mathrm{bpm})$ & 65 & 73 & 58 & 78 & 72 & 60 & 75 & 88 \\
\hline \hline
\end{tabular}

\section{A. Surface EMG, Amount of Energy Consumption, and Motion Strength Test Method}

Both the early and current Agricultural PAS units were used in this test. The test subjects were healthy adults, including six men and two women. Table I shows the test subjects' body characteristics. Informed consent was obtained from the test subjects at the time of measurements.

\section{B. Measurement of the Surface EMG}

The measurement positions for the surface muscle potential were the shoulder deltoid muscle, where load was applied in shelf work, the vastus medialis muscle and vastus lateralis muscle in the thigh area, and the rectus femoris muscle. The test was performed targeting test subjects A to D. The test subjects took a chair sitting position and performed isometric knee stretching movements for $5 \mathrm{~s}$ at a knee flex angle of $90^{\circ}$ for the measurement of the muscle potential in the MVC of the thigh muscle. They also performed MVC in the arm raised direction, with their arms fixed in a position parallel to the ground, for the measurement of the muscle potential of the deltoid muscle.

The surface muscle potential during the actions simulating work in harvesting shelf crops was then measured. Three grape mockups were positioned at $1 \mathrm{~m}$ intervals centered at about the line-of-sight height to recreate the actual harvest environment for shelf crops. A string was stretched out at a position $20 \mathrm{~cm}$ lower than the line of sight. The subjects grasped the grape mockups with their hands and bent their knees downward in a half-sitting posture until their line-of-sight was at the string for $15 \mathrm{~s}$, then moved for $5 \mathrm{~s}$ toward the front of the adjacent grape mockup position. A total of 15 sets were performed in succession, with $15 \mathrm{~s}$ of the half-sitting posture and $5 \mathrm{~s}$ movement as one set. The surface muscle potential was measured when the subjects were in a half-sitting posture. The test was performed when the Agricultural PAS was not worn, when the previous suit was worn, and when the current suit was worn. Fig. 3 shows the actual appearance.

The personal EMG made by Oisaka Electronic Equipment Ltd. was used to measure the surface muscle potential. Meanwhile, the Blue Sensor M Electrode made by Ambu A/S was used for the electrode. The surface muscle potential value was obtained through a band pass filter with a sampling frequency of $3 \mathrm{kHz}$, an amplitude of $1000 \times$, and a frequency interval of 10 to $300 \mathrm{~Hz}$.

\section{C. $\mathrm{VO}_{2}, \mathrm{VCO}_{2}$, and Heart Rate Measurement}

The test was performed by targeting all the test subjects. As for the measurement of the surface muscle potential, the test subjects performed a half-sitting posture for $15 \mathrm{~s}$, moved for $5 \mathrm{~s}$, then repeated these two actions for 15 times. Subsequently, the oxygen intake volume $\mathrm{VO}_{2}$, carbon dioxide discharge volume $\mathrm{VCO}_{2}$, and heart rate $\mathrm{HRR}$ were measured for $5 \mathrm{~min}$. The test was performed when the Agricultural PAS was not worn, when the previous suit was worn, and when the current suit was worn.

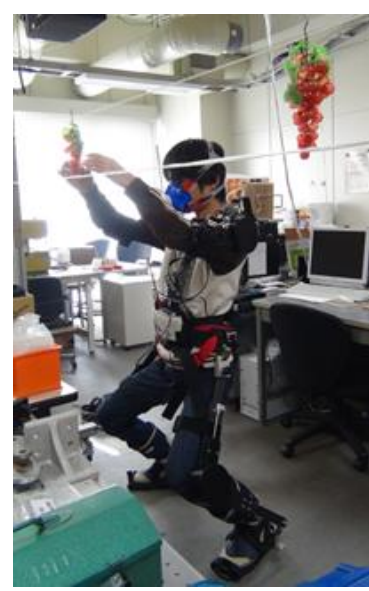

Fig. 3. Experiment simulating berry thinning.

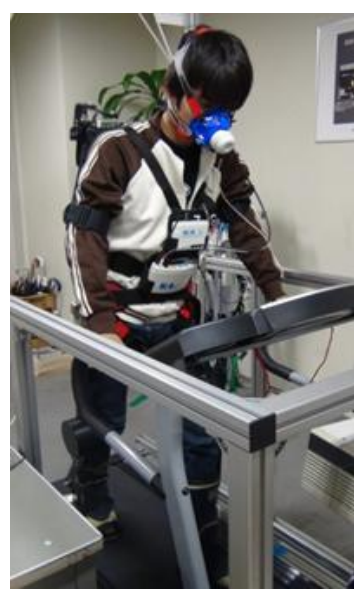

Fig. 4. Walking experiment.
A walking test was also performed assuming movement in a farm field. For the test, the subjects walked on a treadmill at a speed of $2 \mathrm{~km} / \mathrm{h}$ for $5 \mathrm{~min}$ (Johnson Health Tech Japan, Trail Jogger T102). Their $\mathrm{VO}_{2}, \mathrm{VCO}_{2}$, and heart rate were then recorded. The $\mathrm{VO}_{2}[\mathrm{ml} / \mathrm{min}], \mathrm{VCO}_{2}[\mathrm{ml} / \mathrm{min}]$, and heart rate $[\mathrm{bpm}]$ were measured using the COSMED K4b2. The test was performed when the Agricultural PAS was not worn, when the current suit was worn, and when the previous suit was worn. Fig. 4 presents the actual appearance.

\section{Test Results and Discussion}

Fig. 5 shows the change related to the normalized test subject B deltoid muscle MPF, with the MPF for the first half-sitting posture measurement set to 100 . The MPF in the deltoid muscle and the rectus femoris muscle when the Agricultural PAS was not worn showed a declining trend, with a maximum decline of approximately $15 \%$. A reduction in fatigue was observed in these cases because no MPF decline was found when the previous and current suits were worn. The same trend was seen even for test subject D. For test subject C, Fig. 6 shows no major change between when the Agricultural PAS was worn and when it was not worn. The same trend was seen for test subject A. The MPF approximation line was then found. Fig. 7 illustrates the average trend for all the test subjects by muscle location. As regards the deltoid and vastus medialis muscles, the subjects experienced more fatigue when the Agricultural PAS was not worn than when the Agricultural PAS was worn because the MPF trend was large. For the vastus lateralis and rectus femoris muscles, the trend was larger when the suit was worn than when not worn. However, no change in fatigue was indicated because the trend was close to 0 .

Fig. 8 depicts the average for all the test subjects in relation to $\% \mathrm{MVC}$ by muscle location. A decrease in the $\% \mathrm{MVC}$ of $21.4 \%, 11.4 \%, 14.6 \%$, and $13.3 \%$ was observed for the deltoid, vastus medialis, vastus lateralis, and rectus femoris muscles, respectively, when the previous Agricultural PAS 
unit was worn. Furthermore, a decrease in the \%MVC of $17.4 \%, 14.0 \%, 18.7 \%$, and $15.5 \%$ was found for the deltoid, vastus medialis, vastus lateralis, and rectus femoris muscles, respectively, when the current Agricultural PAS unit was worn.

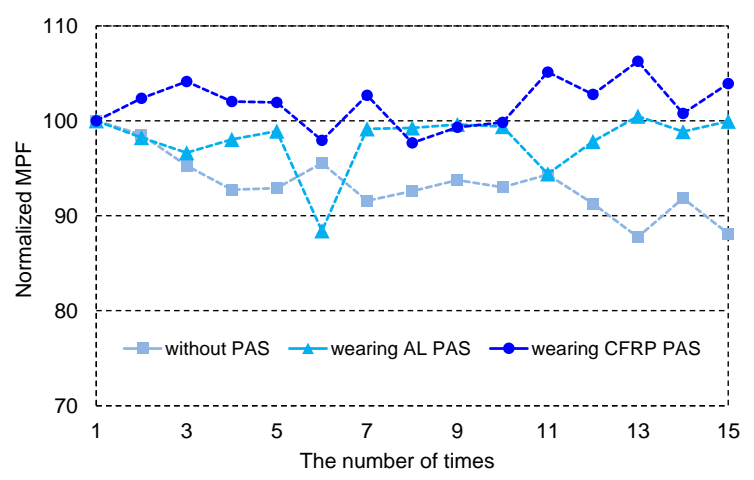

Fig. 5. Normalized MPF of the deltoid muscle of subject B.

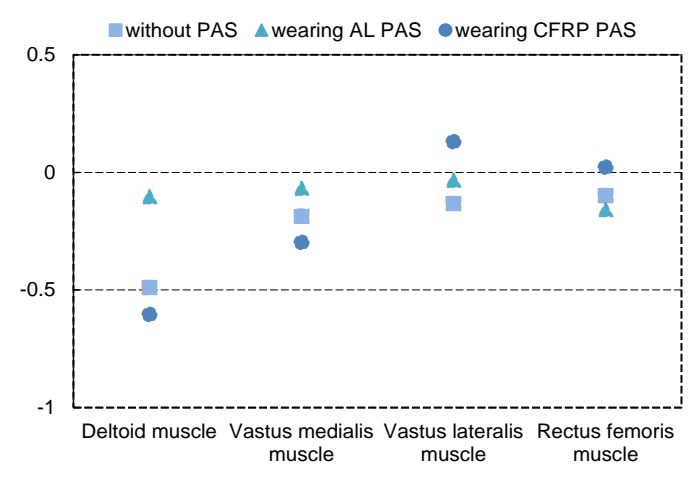

Fig. 7. Normalized MPF.

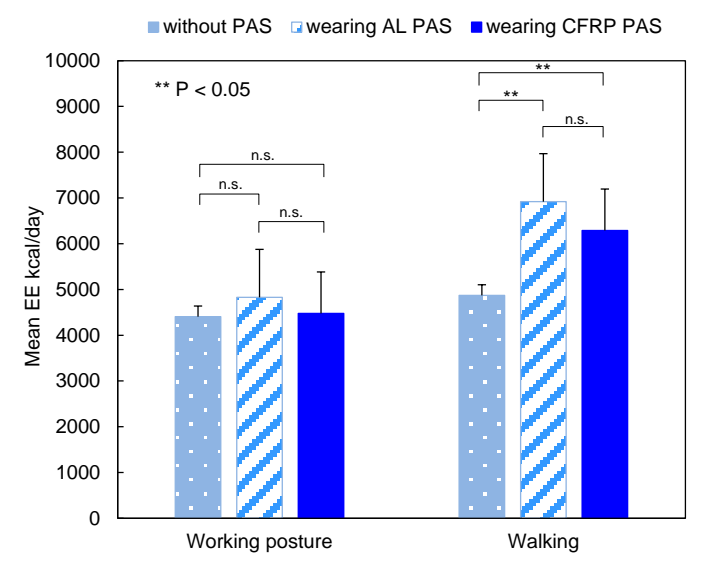

Fig. 9. Normalized MPF.

The current suit \%MVC was lower than that of the previous suit in the three muscles of the thigh because the Agricultural PAS weight itself during the work stance was supported through the foot frame to the floor via the spiral spring. In addition, a reduction in the Agricultural PAS weight appeared to have had an effect of relatively increasing the assist force in the knee joint area.

Fig. 9 shows the average for all the test subjects in relation to the EE. The previous suit showed the highest $\mathrm{EE}$ value in both the work and the walking stance. Compared with when the Agricultural PAS was not used, the EE increased by 423 $\mathrm{kcal} /$ day during the work stance and by $2050 \mathrm{kcal} /$ day during the walking stance when the previous suit was worn. One reason for this result is that many of the test subjects were wearing the Agricultural PAS for the first time and were not familiar with its operation.

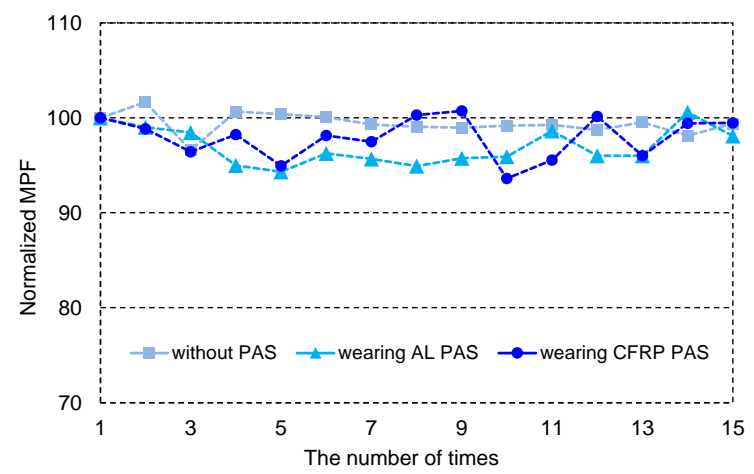

Fig. 6. Normalized MPF of the vastus lateralis muscle of subject C.

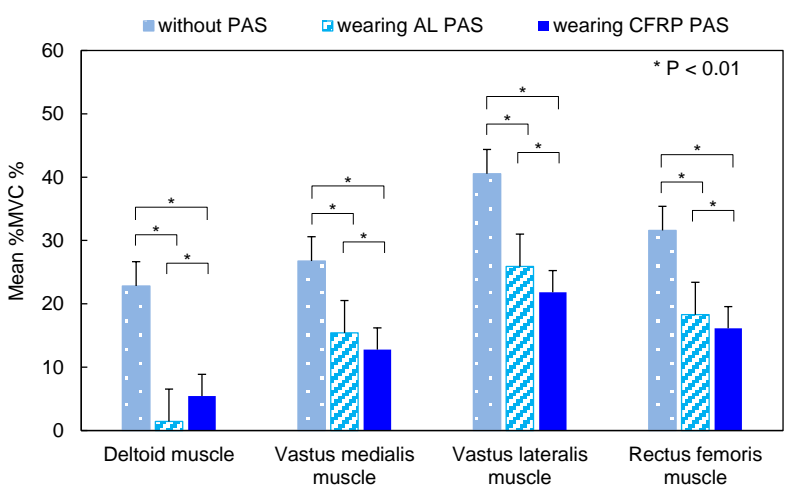

Fig. 8. Mean \%MVC.

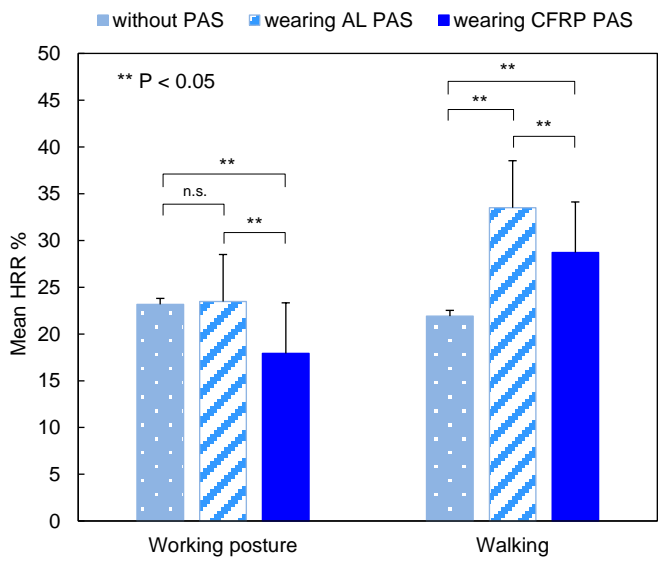

Fig. 10. Mean \% MVC.

In addition, the subjects appeared to have felt the weight of the Agricultural PAS itself because they were unfamiliar with movement while wearing the Agricultural PAS. Meanwhile, the EE when the current Agricultural PAS unit was worn was approximately the same as that when no PAS was worn for a work stance, but it was greater by $1420 \mathrm{kcal} /$ day when walking. However, the EE when the current suit was worn was $630 \mathrm{kcal} /$ day less than that when the previous suit was worn. Therefore, the load on the wearer appeared to be improved by the lighter weight of the current suit.

Fig. 10 presents the average for all the test subjects in 
relation to the HHR. The current suit had the lowest HHR value during the work stance. Compared to the case when the Agricultural PAS was not worn, a decrease of $5.0 \%$ in the HHR was demonstrated, thereby indicating that the work stance load could be reduced. As regards the EE, the weight of the previous Agricultural PAS itself was a load on the wearer when moving and had the highest HHR value during the work stance. When walking, an increase of $11.6 \%$ in the HHR was observed for the previous suit compared to when the Agricultural PAS was not worn. Meanwhile, an increase of $6.8 \%$ was found for the current suit. When walking, the HHR appeared to increase because of the effect of the weight of the Agricultural PAS itself. A comparison of the results obtained for the early and current suits showed that the load on the wearer was improved in the current suit because of its lighter weight.

\section{CONCLUSION}

The labor-reducing effect of the previously developed Agricultural PAS for shelf crop thinning and harvesting work was verified in this study. Accordingly, quantitative measurements were made during the simulated labor work reducing effect indices, including those of the surface muscle potential, amount of energy consumption, and motion strength. These measurements indicated that while the previous suit exhibited a large effect in a static action (e.g., holding a stance), its own weight was a load on the wearer in a dynamic action (e.g., movement). Compared with the previous suit, the current suit showed potential for reduced labor in a static action because of its reduced weight. In addition, the suit improved the freedom of knee movement in a dynamic action and achieved the effect of reducing its own weight load. This work showed that the current suit has a greater labor-reducing effect in shelf crop harvesting or thinning work while considering the degree of learning that the wearer would require to operate it.

\section{REFERENCES}

[1] M. Sato and E. Yagi, "A study on power assist suit using pneumatic actuators based on calculated retaining torques for lift-up motion," in Proc. of SICE Annual Conference, pp. 628-632, 2011.

[2] K. Sano, E. Yagi, and M. Sato, "Development of a wearable assist suit for walking and lifting-up motion using electric motors," Journal of Robotics and Mechatronics, vol. 25, no. 6, pp. 923-930, 2013.

[3] G. Yamamoto and S. Toyama, "Development of wearable-agri-robot $\sim$ mechanism for agricultural work ," in Proc. International Conference on Intelligent Robots and System, pp. 5801-5806, 2009.

[4] S. Toyama and G. Yamamoto, "Wearable agrirobot," Journal of Vibroengineering, vol. 12, pp. 287-291, 2010.

[5] Y. Y. Nwe et al., "Workload assessment with Ovako working posture analysis system (OWAS) in Japanese Vineyards with focus on pruning and berry thinning operations," J. Japan. Soc. Hort. Sci, vol. 81, no. 4, pp. 320-326, 2012.

[6] S. P. Arjunan, D. K. Kumar, and G. Naik. (2014). Computation and evaluation of features of surface electromyogram to identify the force of muscle contraction and muscle fatigue. BioMed Research International. $\quad$ [Online]. $2014 . \quad$ Available: http://dx.doi.org/10.1155/2014/197960.
[7] S. J. Strath et al., "Evaluation of heart rate as a method for assessing moderate intensity physical activity," Medicine \& Science in Sports \& Exercise, vol. 32, pp. S465-S470, 2000.

[8] A. Tajima, "Assessment of energy expenditure: An association between heart rate and oxygen uptake in daily physical activity," International Journal of Analytical Bio-Science, vol. 2, no. 4, pp. 135-142, 2014.

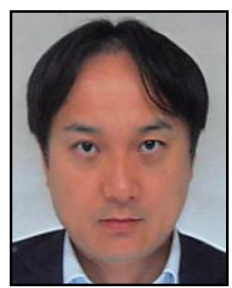

Toshitake Araie was born in Kaga of Japan, Sept. 19, 1975. He is 3rd year student in the doctoral program of Tokyo University of Agriculture and Technology. $\mathrm{He}$ is assistant professor of faculty of human resources development at the Polytechnic University. His primary research interests include development of agricultural power assist suit, life support technology and embodied cognitive science.

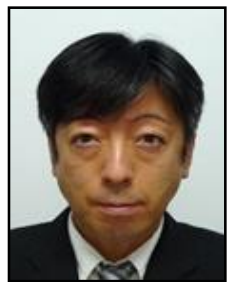

Uichi Nishizawa was born in Kodaira of Japan, 1970 He received his Ph.D degrees from the Tokyo University of Agricultural and Technology. He is assistant professor of faculty of Engineering Mechanical Systems Engineering at the Tokyo University of agriculture and Technology. His primary research interests include robotics, aerospace engineering, and welfare technology.

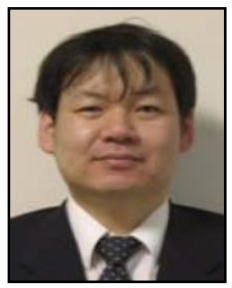

Tomozumi Ikeda was born in Matsumoto of Japan, Aug. 1, 1971. He received his Ph.D degrees from the University of Electro-Communications in 2007. He is associate professor of faculty of human resources development at the Polytechnic University. His primary research interests include life support technology, embodied cognitive science and development of multimodal interface for visually impaired people.

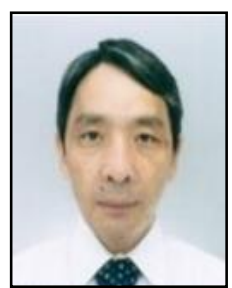

Akira Kakimoto was born in Hiroshima, Jan. 10, 1962. He graduated from the University of Tokyo in department of precision machinery engineering in faculty of engineering, received master's degree in 1986 and doctor's degree in 1990 in department of precision machinery engineering in the graduate school of engineering in the University of Tokyo. Research associate in department of rehabilitation engineering in the institute of vocational training (presently, the Polytechnic University) in 1990. He is the assistant professor in department of mechanical and control engineering in 1992, professor in mechanical system engineering in 2010. Currently he is interested in assistive technology and machine control.

Prof. Kakimoto, member of EMBS IEEE, JSPE, JSME, LST, JSWSAT.

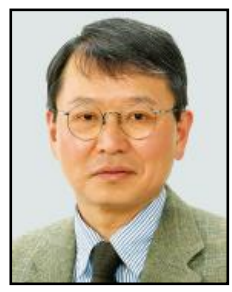

Shigeki Toyama was born in Gifu of Japan, 1954. He graduated from the University of Tokyo in department of precision machinery engineering in faculty of engineering, received doctor's degree in 1981 in department of precision machinery engineering in the graduate school of engineering in the University of Tokyo. He is professor of faculty of Engineering Mechanical Systems Engineering at the Tokyo University of agriculture and Technology. His primary research interests include robotics, actuator, and welfare technology. 